\title{
Comparison of breast reconstruction using ipsilateral and contralateral pedicle transverse rectus abdominis musculocutaneous flaps
}

\author{
Ramesh Omranipour, Sima Mashayekhi, Mahtab Vasigh, Sadaf Alipour, Habibollah Mahmoodzadeh
}

Cancer Institute, Tehran University of Medical Sciences, Imam Khomeini Hospital, Tehran 13145-158, Iran.

Correspondence to: Prof. Ramesh Omranipour, Cancer Institute, Tehran University of Medical Sciences, Imam Khomeini Hospital, Tehran 13145158, Iran. E-mail: omranipour@tums.ac.ir

How to cite this article: Omranipour R, Mashayekhi S, Vasigh M, Alipour S, Mahmoodzadeh H. Comparison of breast reconstruction using ipsilateral and contralateral pedicle transverse rectus abdominis musculocutaneous flaps. Plast Aesthet Res 2017;4:155-60.

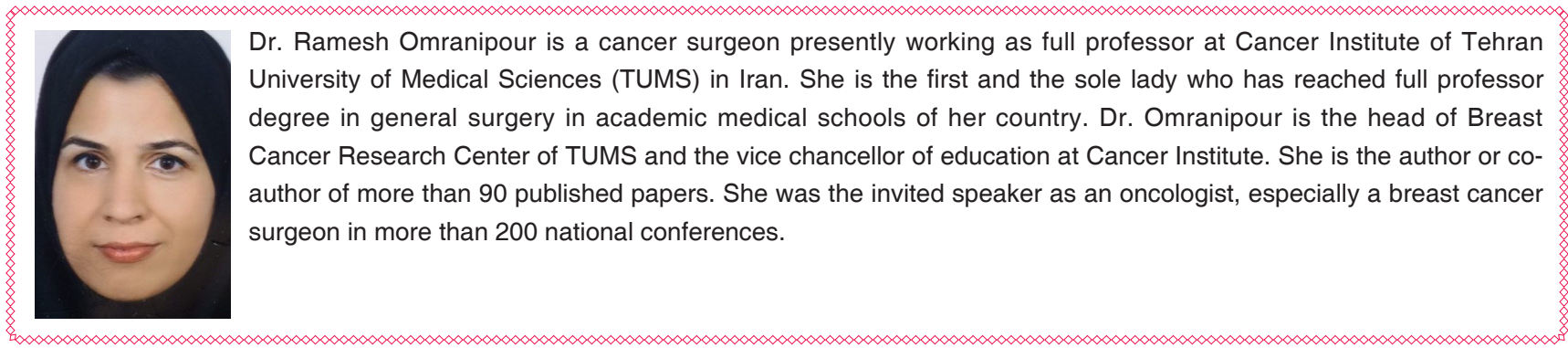

Article history:

Received: 17 Aug 2017

Accepted: 19 Sep 2017

Published: 29 Sep 2017

\section{Key words:}

Breast reconstruction, transvers rectus abdominis musculocutaneous flap, unilateral pedicle TRAM flap, ipsilateral pedicle TRAM flap

\begin{abstract}
Aim: Breast reconstruction has several beneficial effects on psychosocial well-being and quality of life. The ultimate goal has always been to create the most natural breast mound. Thus in many centers, the unilateral pedicled transverse rectus abdominis myocutaneous (TRAM) flap remains the most common technique for breast reconstruction. Our objective was to retrospectively compare the outcomes of ipsilateral and contralateral pedicle TRAM flaps. Methods: The total of 110 patients underwent unilateral breast reconstruction with pedicle TRAM flap at Cancer Institute of Tehran University of Medical Science from January 1996 to June 2011. Premorbid risk factors, postoperative outcomes and demographic data were assessed. The analysis of the recordings was done by SPSS 20. Results: Out of 110 patients who were included in the study, 87 had ipsilateral and 23 contralateral pedicle TRAM flaps. The incidence of flap complications that did not require surgical intervention was $19.7 \%$ in ipsilateral and $30.4 \%$ in contralateral pedicle TRAM flap. The incidence of flap loss requiring revision was significantly higher in contralateral group $(P=0.001)$. Major complications were noted in $11.5 \%$ of the ipsilateral pedicle TRAM patients and $26.1 \%$ of the contralateral group $(P<0.001)$. Minor complications were noted in $17.2 \%$ of the ipsilateral
\end{abstract}

cc) (1) 5 This is an open access article distributed under the terms of the Creative Commons Attributioncc) NC SA NonCommercial-ShareAlike 3.0 License, which allows others to remix, tweak, and build upon the work non-commercially, as long as the author is credited and the new creations are licensed under the identical terms. 
pedicle TRAM patients and in $34.8 \%$ of the contralateral group $(P<0.001)$. Total early hospital stay was longer in contralateral pedicle TRAM flaps (7.66 days vs. 10.68 days, $P=0.83$ ). Higher complications were encountered in contralateral pedicle TRAM flaps compared to ipsilateral pedicle TRAM patients $(39.1 \% v s .19 .5 \%, P=0.001)$. The type of pedicled TRAM flap (ipsilateral $v s$. contralateral), had significant effect on complications (odds ratio $=0.007, P=0.002$ ) while other variables had no significant effect on the incidence of complications. Conclusion: This study indicates that the overall outcome and mid-term morbidity-free survivals of ipsilateral pedicle TRAM flap breast reconstruction are statistically superior to contralateral pedicle TRAM flap breast reconstruction. Both of these procedures are reasonably feasible and safe. These findings lead us to discourage the use of contralateral pedicle TRAM flap when an ipsilateral option is feasible.

\section{INTRODUCTION}

Breast reconstruction has several beneficial effects on the psychosocial well-being and quality of life. Different studies have shown that breast reconstruction improves self-image, sexuality, and decreased rates of depression in women who have had mastectomy ${ }^{[1]}$. Additionally, patients who undergone reconstruction with autologous tissue, in comparison with those who have undergone reconstruction with tissue expanders/ implants, have better long-term quality of life ${ }^{[2]}$.

Transverse rectus abdominis myocutaneous (TRAM) techniques have long been applied but selecting a superior technique is controversial. The selection of the best procedure significantly affects the outcome of flap viability, patients' satisfaction, and quality of life $^{[3]}$. The pedicle TRAM flap is still one of the most common procedure performed in many centers. Our study demonstrates that it is associated with a low complication rate and a high level of patient satisfaction in our center ${ }^{[4]}$.

Morbidity and adverse events following different reconstructive breast surgeries are reported to be widely varied. In general, patients selected for pedicle TRAM flap reconstruction must have adequate abdominal soft tissue for the procedure to be successful. There are several identified risk factors contributing to the post operative complications of pedicle TRAM flap reconstruction. These factors are cigarette smoking, obesity, prior radiation therapy, abdominal surgery, and significant medical comorbidities $^{[5]}$.

As the experience and comfort with micro-surgical techniques and instrumentation and post-op monitoring facilities are not available in all centers. Surgeons perform the pedicle TRAM flap because it demands less technical and facility requirements and has fewer complete flap loss in comparison with free flap techniques ${ }^{[6]}$.

Rotation of pedicle TRAM flap can be ipsilateral or contralateral. Some surgeons believe that the ipsilateral procedure might result in more tension on the vascular pedicle and folding of the pedicle could reduce the blood supply of the flap ${ }^{[7,8]}$. In order to avoid the folding and the vascular flow surgeons have preferred to use the contralateral pedicle TRAM flap. The contralateral pedicle TRAM flap also seemed to have some aesthetic limitations due to ablation of the xiphoid subunits and the medial infra-mammary fold. There are also some limitations from the shorter pedicle length ${ }^{[7]}$. There are reports indicating differences in early and long-term outcome of these two techniques ${ }^{[9]}$. Some authors reported similar safety of both techniques ${ }^{[10]}$ while others favored one pedicle TRAM technique over the other on $e^{[8,9]}$.

Our objective was to retrospectively compare outcomes of ipsilateral and contralateral pedicle TRAM flaps in a retrospective cohort study. In our center free flaps for breast reconstruction are not routinely performed. Therefore we performed this study to analyze the outcomes and complications of TRAM flaps performed in our center. We were able to assess the risk factors which could cause more post-operative complications and compare the results of ipsilateral and contralateral TRAM flaps retrospectively. We are aware that free flaps will substitute TRAM flaps inevitably, so in this study we measured the results of TRAM flaps in our center. The study primary endpoints were postoperative morbidity (defined as occurrence of at least one of the postoperative complications within the follow-up period), need for re-hospitalization and need for re-operation.

\section{METHODS}

In total of 110 patients who underwent unilateral breast reconstruction with pedicle TRAM flap at the Cancer Institute of Tehran University of Medical sciences from January 1996 to June 2011, were included in this study. The ethics committee accepted this study and the patients were informed about it during their follow-up exams. Patients with micro-vascular supercharging of the flap or those who received a bi-pedicle or bilateral TRAM flap were excluded. Patients' age, height, weight, history of smoking, and associated comorbidities (diabetes mellitus, dyslipidemia and hypertension), steroid use, history of liposuction, tumor staging (based on TNM criteria), presence of tumor markers (estrogen receptor status, progesterone 
receptor, P53, Her2Neu), history of post-mastectomy radiotherapy or chemotherapy and finally the time length between mastectomy and reconstruction were abstracted from the medical records. Type of pedicle TRAM (ipsilateral vs. contralateral), timing of the procedure (immediate vs. delayed), and usage of mesh for the abdominal wall closure were also recorded.

All patients had follow-up examinations every week for the first month and monthly thereafter for 6 months. Postoperative outcomes were assessed using clinical data records if available, otherwise we communicated with the patients' physicians. The mean follow-up duration was 2.69 years for ipsilateral group and 3.21 years for contralateral group.

Immediate post-reconstructive complications were recorded. Postoperative complications were then categorized into major or minor events. Complications which required re-admission or re-hospitalization such as total flap loss were defined as complete necrosis of the skin and fat; partial flap loss, was defined as ischemic tissue loss exceeding $25 \%$ of the flap. Wound infection was defined as redness, swelling, and exudate and requiring antibiotics. Seroma formation was defined as palpable fluctuation of subcutaneous tissues requiring suction or drainage. Hematoma, requiring evacuation, pulmonary embolism, sepsis, hernia, ileus and acute renal failure were categorized as major complications. All the other complications were categorized as minor.

SPSS 20 (SPSS Inc., Chicago, IL, USA) was used for statistical analysis, with comparison of discrete variables by Pearson chi-square or Fisher's exact test, and comparison of means by $t$-test. A value of $P<$ 0.05 was considered significant. Predictors exhibiting a statistically significant relation with postoperative morbidity were taken for multivariate logistic regression analysis to evaluate their independence as predictors. Odds ratio (OR) and 95\% confidence intervals were calculated.

\section{RESULTS}

Table 1 presents the baseline characteristics of the study population and the type of procedures. Out of 110 patients who were included in the study, 87 had ipsilateral and 23 contralateral pedicle TRAM flaps. There were no significant differences in demographic characteristics between ipsilateral and contralateral pedicle TRAM flap groups. Tumor size and the number of lymph node involvement were higher in contralateral pedicle TRAM flap group. Immediate reconstruction was more commonly performed in contralateral pedicle TRAM flap group, $(34.8 \%$ vs. $10.3 \%$, respectively).

Co-morbidities included hypertension, diabetes mellitus, hyperlipidemia, presence of abdominal scar, and smoking. The two procedures had similar clinical and demographic characteristics. Co-morbidities were more common in ipsilateral pedicle TRAM group.

Table 2 summarizes the categories of complications during follow-up period. Comparing the ipsilateral with the contralateral TRAM groups, the incidence of flap complications that did not require surgical intervention was $19.7 \%$ in ipsilateral and $30.4 \%$ in contralateral pedicle TRAM flap, respectively. The incidence of flap loss requiring revision was significantly higher in contralateral group $(P=0.001)$. Major complications (any complication that required hospital admission or operative procedure) were noted in $11.5 \%$ of the ipsilateral pedicle TRAM patients and $26.1 \%$ of the contralateral group $(P<0.001)$. Minor complications were noted in $17.2 \%$ of the ipsilateral pedicle TRAM patients and in $34.8 \%$ of the contralateral group, respectively $(P<0.001)$. When individual complications

Table 1: Baseline characteristics of the study population and the type of procedures for breast reconstruction, $n$ (\%)

\begin{tabular}{lccc}
\hline Characteristics & $\begin{array}{c}\text { Ipsilateral pedicled } \\
\text { TRAM }(\boldsymbol{n = 8 7})\end{array}$ & $\begin{array}{c}\text { Contralateral pedicled } \\
\text { TRAM }(\boldsymbol{n}=\mathbf{2 3})\end{array}$ & $\boldsymbol{P}$ \\
\hline Age $($ years), mean $\pm \mathrm{SD}$ & $42.58 \pm 9.15$ & $39.22 \pm 6.6$ & 0.99 \\
Body mass index $\left(\mathrm{kg} / \mathrm{m}^{2}\right)$, mean $\pm \mathrm{SD}$ & $27.43 \pm 4.28$ & $24.54 \pm 3.47$ & 0.64 \\
Diabetes mellitus & $9(10.1)$ & $1(4.3)$ & 0.003 \\
Hypertension & $6(6.9)$ & $1(4.3)$ & 0.002 \\
Hyperlipidemia & $1(1.1)$ & $0(0.0)$ & 0.011 \\
Tobacco use & $7(8)$ & $1(4.3)$ & 0.005 \\
Abdominal scar & $22(25.3)$ & $11(47.8)$ & 0.004 \\
History of radiotherapy & $65(74.7)$ & $12(52.2)$ & 0.025 \\
History of chemotherapy & $61(70.9)$ & $19(82.6)$ & 0.67 \\
Immediate reconstruction & $9(10.3)$ & $8(34.8)$ & 0.001 \\
Time between mastectomy and reconstruction & $50.39 \pm 42.14$ & $36.51 \pm 30.65$ & 0.071 \\
\hline
\end{tabular}

TRAM: transverse rectus abdominis myocutaneous 
Table 2: Postoperative complications of ipsilateral and contralateral pedicle TRAM flap, $\boldsymbol{n}(\%)$

\begin{tabular}{lccc}
\hline Characteristics & $\begin{array}{c}\text { Ipsilateral pedicled } \\
\text { TRAM }(\boldsymbol{n = 8 7 )}\end{array}$ & $\begin{array}{c}\text { Contralateral pedicled } \\
\text { TRAM }(\boldsymbol{n}=\mathbf{2 3})\end{array}$ & $\boldsymbol{P}$ \\
\hline Length of stay in hospital (days), mean \pm SD & $7.66 \pm 7.27$ & $10.68 \pm 7.25$ & 0.83 \\
Total morbidity & $17(19.5)$ & $9(39.1)$ & 0.001 \\
Flap ischemia & $1(1.1)$ & $0(0)$ & 0.23 \\
Flap necrosis & $7(8)$ & $4(17.4)$ & 23 \\
Sub-flap hematoma & $0(0.0)$ & $2(8.7)$ & 0.001 \\
Sub-flap seroma & $4(4.6)$ & $5(21.7)$ & 0.040 \\
Flap wound infection & $2(3.3)$ & $4(17.45)$ & 0.059 \\
Deep vein thrombosis & $1(1.1)$ & $1(4.3)$ & 0.74 \\
Re-hospitalization & $7(8)$ & $5(21.75)$ & 0.20 \\
Re-operation & $6(6.9)$ & $4(17.4)$ & 0.35 \\
\hline
\end{tabular}

TRAM: transverse rectus abdominis myocutaneous

were compared by procedure group, sub-flap hematoma, sub-flap seroma, flap necrosis, and flap wound infection were significantly higher in contralateral pedicle TRAM patients.

As summarized in Table 2, the total early hospital stay was longer in contralateral pedicle TRAM flaps. (7.66 days vs. 10.68 days, $P=0.83$ ). There were higher complications in contralateral pedicle TRAM flaps $(39.1 \%$ vs. $19.5 \%, P=0.001)$. Flap necrosis and sub-flap seroma were two most common early postoperative complications in both groups.

Logistic regression was used to assess the effect of procedure technique (ipsilateral vs. contralateral) on major, minor and ischemic flap complications while controlling for patient age, body mass index (BMI), radiation therapy, procedure timing, surgical delay of the flap, comorbidities, smoking and abdominal scar [Tables 3 and 4].

The type of pedicle TRAM flap (ipsilateral vs. contralateral), had significant effect on complications (OR $=0.007, P=0.002)$. Other variables had no significant effect on the incidence of complications.

\section{DISCUSSION}

Although the pedicle TRAM flap provided a foundation for the burgeoning field of breast reconstruction, the overall contemporary trend has focused on approaches which provide improved aesthetic outcomes while minimizing complications and donor site morbidity. The most advantageous benefit of pedicle TRAM flaps as a method of autogenous reconstruction is to employ removed excess lower abdominal tissue thorough a cosmetic abdominoplasty and achieve a long lasting satisfactory outcome. To do this, careful selection of patients and the best procedural technique in addition to pre-operative risk profile management can effectively reduce postoperative adverse events.
A history of mastectomy, chest wall radiation, advanced age, tobacco use, and some other underlying medical conditions are identified as predisposing factors to postoperative complications ${ }^{[5]}$. In our study, based on conclusions from the multivariable regression model, none of the study variables other than the laterality of the flap could predict morbidity.

The overall rate of morbidity observed in our study regardless of the type of technique was $27.4 \%$. This

Table 3: Multivariate analysis of correlation of overall complication with pedicle TRAM breast reconstruction

\begin{tabular}{lcc}
\hline Independent variable & OR $\mathbf{( 9 5 \% ~ C l )}$ & $\boldsymbol{P}$ \\
\hline Type of technique (ipsilateral & $0.007(0.005-0.443)$ & 0.002 \\
vs. contralateral) & 0.98 & 0.49 \\
Age & 0.47 & 0.94 \\
Body mass index & 0.98 & 0.49 \\
Surgical delay of flap & $0.38(0.54-4.92)$ & 0.38 \\
Presence of at least one co- & $0.74(0.127-18.32)$ & 1.644 \\
morbidity & $0.45(0.03-4.55)$ & 0.57 \\
Abdominal scar & $0.12(0.17-1.23)$ & 0.27 \\
Smoking & $0.23(0.68-4.99)$ & 0.24 \\
History of radiotherapy & \\
Timing (immediate vs. &
\end{tabular}

TRAM: transverse rectus abdominis myocutaneous; $\mathrm{Cl}$ : confidence interval; OR: odds ratio

Table 4: Multivariate analysis of correlation of minor complication with pedicle TRAM breast reconstruction

\begin{tabular}{lcc}
\hline Independent variable & OR $(95 \% \mathrm{Cl})$ & $\boldsymbol{P}$ \\
\hline Ipsilateral vs. contralateral & $0.001(0.005-0.38)$ & 0.005 \\
Age & 0.49 & 0.99 \\
Body mass index & 0.39 & 0.8 \\
Surgical delay of flap & 0.42 & 0.85 \\
Presence of at least one co- & $0.11(0.87-14.32)$ & 0.1 \\
morbidity & $0.98(0.28-4.59)$ & 0.87 \\
History of radiation therapy & $0.24(0.58-9.37)$ & 0.23 \\
Timing (immediate vs. & delayed)
\end{tabular}

TRAM: transverse rectus abdominis myocutaneous; $\mathrm{Cl}$ : confidence interval; OR: odds ratio 
seemed to be lower than the previously reported $31.82 \%$ by Fathi et al. ${ }^{[11]}$ The difference might be explained by the differences in the definition of postoperative morbidity and the time of follow-up period in our study in comparison with theirs. We did not encounter any total flap loss which is the most serious complication of microsurgical breast reconstruction.

Unlike some other previous reports we did not find any correlation between age or BMI and morbidity in this study. The only comorbidity which could increase the rate of post-operative complications was smoking.

In this study we aimed to determine the superiority of ipsilateral or contralateral pedicle TRAM flap based on postoperative complications and morbidity. There were differences in total morbidity, flap ischemia, flap necrosis, sub-flap hematoma, sub-flap seroma, and flap infection, between the two pedicle TRAM flap groups. The rate of complications in contralateral pedicle TRAM flaps $(39.1 \%)$ was significantly higher than ipsilateral pedicle TRAM flaps $(19.5 \%),(P=0.001)$. The type of pedicle TRAM flap did affect the total postoperative morbidity, even in the absence of other base line risk factors and co-morbidities. Our results were contrary to some previous reports which indicated that the type of pedicle TRAM flap does not increase postoperative complications. In Clugston et al. ${ }^{[8]}$ study of ipsilateral pedicle TRAMs, the authors reported a moderately high minor complication rate but a relatively low major complication rate ${ }^{[12]}$.

The rate of complications of pedicle TRAM flap in our study $(27.4 \%)$ is similar to other studies $(16-41 \%)^{[13-16]}$. Partial flap necrosis in our study was $13.7 \%$ which is less than previous reports by Janiga et al. ${ }^{[9]}(16-41 \%)$.

We found that the rate of major complications (needing re-hospitalization or re-operation) following pedicle TRAM flap was $17.9 \%$, while the rate of a minor complication was $24.8 \%$. Minor complications included wound infection, seroma or hematoma not requiring operation, and flap ischemia. Major complications, were significantly higher in our contralateral group. This finding can be explained by limitations of contralateral technique. Contralateral pedicle TRAM flap seems to have some aesthetic limitations due to ablation of the xiphoid subunits and the medial infra-mammary fold. There are also some limitations from the shorter pedicle length.

In a previously irradiated breast, because of the ischemia due to damage to the internal mammary vessels, some surgeons prefer not to use ipsilateral pedicle
TRAM flap for breast reconstruction. We could not find any correlation between previous radiotherapy and morbidities after contralateral or ipsilateral pedicle TRAM flap. This result is similar to the study of Janiga et al. ${ }^{[9]}$

The experience gained in these procedures during the last two decades has enabled surgeons to identify certain risk factors such as obesity, previous abdominal surgery, advanced age, and tobacco use that can increase complication rates. Some researchers suggest considering those factors as contra-indications for a pedicle TRAM flap ${ }^{[15,16]}$. We did not find in our study a higher morbidity rates associated with these risk factors except for smoking. The outcome of pedicle TRAM flap reconstruction in obese patient was similar to non-obese patients in our previous report ${ }^{[17]}$.

The most important finding in this study is that it is not necessary to use contralateral TRAM flap to overcome the limitations of ipsilateral TRAM flap. Moreover, we found out more complications in contralateral TRAM flaps.

The present study had some limitations. It is a retrospective study, inevitably it could have confounding biases due to lack of information on some factors not available in the medical records. Patients in this study were operated on by different surgeons and this could affect the outcome of procedures. However, we were successful in comparing the outcomes and morbidityfree survival rates of the two commonly performed pedicle TRAM flaps (ipsilateral vs. contralateral).

This study indicates that the overall outcome and mid-term morbidity-free survivals of ipsilateral pedicle TRAM flap breast reconstruction are statistically superior to contralateral pedicle TRAM flap breast reconstruction. Both of these procedures are reasonably feasible and safe. According to our findings we recommend against the use of contralateral pedicle TRAM flap to overcome limitations of ipsilateral pedicle TRAM flap. Larger prospective studies are required to address this conclusion. We believe that the ipsilateral compared to the contralateral pedicle TRAM flap has noticeable advantages including total flap vascularity, seroma formation, and partial flap necrosis. In the end, a surgeon's familiarity and experience with either procedure, is likely the most important predictor of a good outcome.

\section{DECLARATIONS}

Authors' contributions

Concept and design: R. Omranipour 
Literature research and manuscript preparation:

S. Mashayekhi, H. Mahmoodzadeh

Data acquisition: S. Alipour

Data analysis and statistical analysis and manuscript editing: M. Vasigh

\section{Financial support and sponsorship None.}

\section{Conflicts of interest \\ There are no conflicts of interest.}

\section{Patient consent \\ Not applicable.}

\section{Ethics approval}

Approved by the ethics committee and the patients were informed about this study during their follow-up period.

\section{REFERENCES}

1. Eltahir Y, Werners LL, Dreise MM, van Emmichoven IA, Jansen L, Werker PM, de Bock GH. Quality-of-life outcomes between mastectomy alone and breast reconstruction: comparison of patientreported BREAST-Q and other health-related quality-of-life measures. Plast Reconstr Surg 2013;132:e201-9.

2. Hu ES, Pusic AL, Waljee JF, Kuhn L, Hawley ST, Wilkins E, Alderman AK. Patient-reported aesthetic satisfaction with breast reconstruction during the long-term survivorship period. Plast Reconstr Surg 2009;124:1-8.

3. Grotting JC, Beckenstein MS, Arkoulakis NS. The art and science of autologous breast reconstruction. Breast J 2003;9:350-60.

4. Omranipour R, Lebaschi AH, Mohagheghi MA, Arab-Kheradmand A, Abasahl A. Outcomes of breast reconstruction with pedicled transverse rectus abdominis myocutaneous (tram) flap at cancer institute, a retrospective study of 10 years of experience. Acta Medica Iranica 2008;46:218-24.

5. Serletti JM. Breast reconstruction with the TRAM flap: pedicled and free. J Surg Oncol 2006;94:532-7.

6. Knox AD, Ho AL, Leung L, Tashakkor AY, Lennox PA, Van Laeken N, Macadam SA. Comparison of outcomes following autologous breast reconstruction using the DIEP and pedicled TRAM flaps: a 12year clinical retrospective study and literature review. Plast Reconstr Surg 2016;138:16-28.

7. Olding M, Emory RE, Barrett WL. Preferential use of the ipsilateral pedicle in TRAM flap breast reconstruction. Ann Plast Surg 1998;40:349-53.

8. Clugston PA, Gingrass MK, Azurin D, Fisher J, Maxwell GP Ipsilateral pedicled TRAM flaps: the safer alternative? Plast Reconstr Surg 2000;105:77-82.

9. Janiga TA, Atisha DM, Lytle IF, Wilkins EG, Alderman AK. Ipsilateral pedicle TRAM flaps for breast reconstruction: are they as safe as contralateral techniques? J Plast Reconstr Aesthet Surg 2010;63:322-6.

10. Ozkan A, Cizmeci O, Aydin H, Ozden BC, Tümerdem B, Emekli $\mathrm{U}$, Asoğlu O, Bozfakioğlu Y. The use of the ipsilateral versus contralateral pedicle and vertical versus horizontal flap inset models in TRAM flap breast reconstruction: the aesthetic outcome. Aesthetic Plast Surg 2002;26:451-6.

11. Fathi M, Hatamipour E, Fathi HR, Zehtab H. Breast reconstruction using tram flap: prospective outcome and complications. MJIRI 2006;20:74-81.

12. Clugston PA, Gingrass MK, Azurin D, Fisher J, Maxwell GP. Ipsilateral pedicled TRAM flaps: the safer alternative? Plast Reconstr Surg 2000;105:77-82.

13. Ascherman JA, Seruya M, Bartsich SA. Abdominal wall morbidity following unilateral and bilateral breast reconstruction with pedicled TRAM flaps: an outcomes analysis of 117 consecutive patients. Plast Reconstr Surg 2008;121:1-8.

14. Tribondeau P, Soffray F. Breast reconstruction with pedicled TRAM flap (a retrospective study of 115 consecutive cases). Ann Chir Plast Esthet 2008;53:309-17. (in French)

15. Shestak KC. Technical tips for avoiding complications in TRAM flap breast reconstruction. In: Reoperative Plastic Surgery of the Breast. PA: Lippincott Williams \& Wilkins; 2006. p. 302-39.

16. Zenn MR, May JW Jr. TRAM flap reconstruction: the single pedicle, whole muscle technique. In: Spear SL, editors. Surgery of the Breast: Principles and Art. PA: Lippincott Williams \& Wilkins; 2006. p. 732-40.

17. Alipour S, Omranipour R, Akrami R. Obesity should not prevent from TRAM flap breast reconstruction in developing countries. Indian $J$ Surg 2015;77:341-4. 